\title{
Amalgamation of Innovation and Elegance in Drug Delivery
}

\section{Shende $P^{*}$ and Sable A}

Shobhaben Pratapbhai Patel School of Pharmacy and Technology Management, Mumbai, India

\begin{abstract}
With the advent of new technology, there are many advances in the field of drug delivery for sustained or controlled release of drug. The conventional drug delivery is undergoing reforms to develop new and innovative drug delivery systems (DDS). The concept of elegant DDS involves the administration of drug or combination of drugs in innovative and attractive way without individuals' acceptable route like administration of antifungal drugs for paronychia using medicated nail lacquer. Other systems involved are medicated lipsticks, medicated tattoos, medicated chewing gum, etc. Also in the era of wearable technology and artificial intelligence, use of wearable's to monitor and diagnose different disease conditions like anaphylaxis and cardiac disease is the area of interest for research. All these wearable's and formulations are used to provide an elegant look as well as therapeutic effect. The present review highlights the present scenario of different elegant DDSs and also focuses on their method of preparation, excipients and also the evaluation parameters. The elegant drug delivery will be the modernised cosmeceuticals for management and treatment of complicated conditions in near future.
\end{abstract}

Keywords: Nail lacquer; Medicated tattoo; Medicated lipstick; Medicated chewing gum

\section{Introduction}

The basic purpose of any DDS is to deliver the drug in a safe and efficacious manner so as to achieve the therapeutic effect in humans and animals [1]. An ideal DDS is the one which increases the bioavailability of the drug when administered in the body provides site-specific action and delivers the drug in a controlled manner in the body (Figure 1).

The other characteristics of an ideal DDS are it must provide delivery of a wide variety of drugs through the same process, high amount of drug dispersion, ease of administration to patients, consistent delivery of drug at various physiological fluids and cost effectiveness [2]. Elegant DDS is a modification of DDS to enhance the behaviour of conventional system in modernized way in consideration with external appearance. Some of the conventional DDS are:

\section{Oral DDS}

\section{Parenteral DDS}

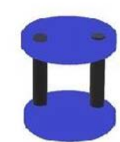

Wearable earring

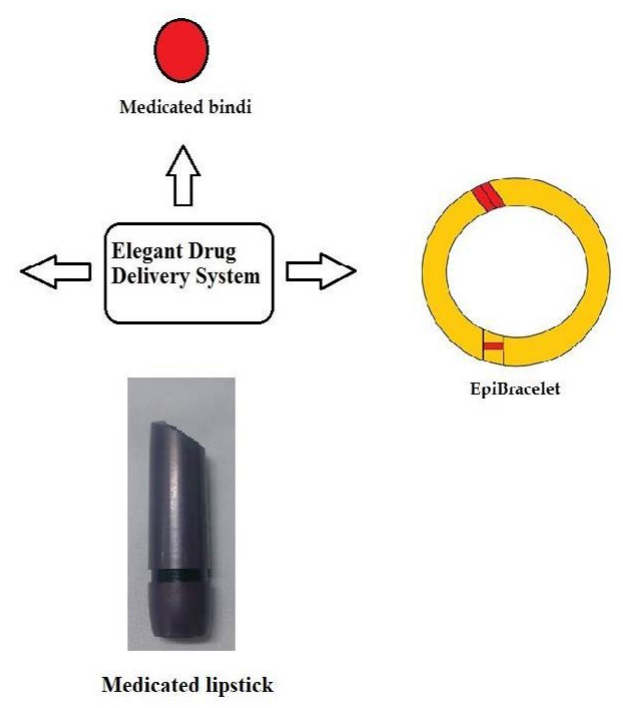

Figure 1: Schematic presentation.
3. Transdermal DDS

4. Nasal DDS

5. Pulmonary DDS

6. Pulsatile DDS

7. Targeted DDS.

Elegant DDS is a combination of science and art with therapeutic potential and beautification purpose. Elegant DDS involves the administration of drugs in an innovative way with the help of cosmetics and wearable devices which can also be used for decorative purposes. Many such examples include medicated nail lacquers, medicated lipsticks and medicated kohl. With the advent of new technologies, the DDS emerge to smart and intelligent DDS. An important aspect of such DDS is to cater to the individual's need for a drug as soon as a physiological change is observed in the patient's body. These smart DDS include the use of smart wearable systems like biosensors, patches, implantable devices etc. [3]. These smart wearable systems can be used as a fashion accessory to modify looks. The advances in the field of these smart devices have made it possible for us to monitor our healthcare anywhere and at any point of time in the form of portable accessories or jewelleries [3]. These devices enable administration of drugs in individuals without making them aware of it (Figure 2) [4].

\section{History}

Elegant DDS includes delivery of drug in an attractive and innovative way for elegance. M De La Cruz was among the first writers to promote the use of chocolate for medicinal purposes in at the Santa Cruz College founded in 1536 [5]. He reported that chocolate could be used for treating diseases like angina, dysentery, gout etc. Then in 1577

*Corresponding author: Shende P, Associate professor, Shobhaben Pratapbhai Patel School of Pharmacy and Technology Management, Mumbai, India, Tel: +9122-42332000; E-mail: Pravin.Shende@nmims.edu

Received March 14, 2018; Accepted March 17, 2018; Published March 28, 2018

Citation: Shende P, Sable A (2018) Amalgamation of Innovation and Elegance in Drug Delivery. J Formul Sci Bioavailab 2: 120.

Copyright: $\odot 2018$ Shende $\mathrm{P}$, et al. This is an open-access article distributed unde the terms of the Creative Commons Attribution License, which permits unrestricted use, distribution, and reproduction in any medium, provided the original author and source are credited. 


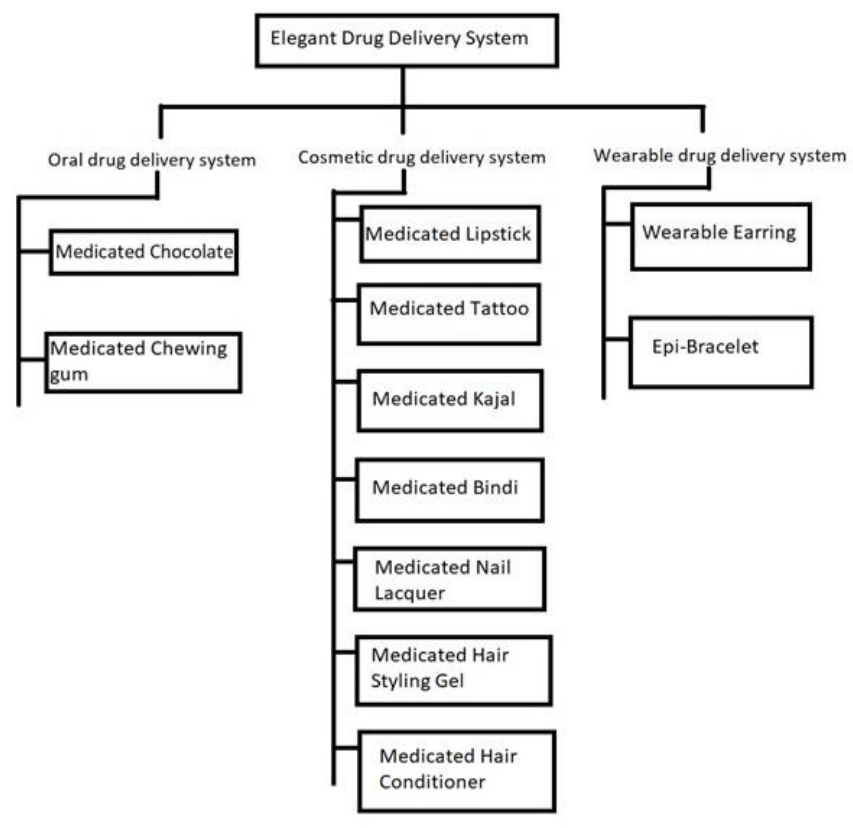

Figure 2: Classification of elegant DDS.

Francisco Hernandez proposed that chocolate could be used to treat liver disease [5]. It has been studied that chewing gum like substances have been used since ancient times but it has a relatively short history. The history of chewing gum dates back to the ancient Egypt and Mayan Indian times when these people used to chew the resin of trees [6]. Medicated chewing gum became known as a marketed product in 1869 when Dr. William F. Semple, a dentist by profession filed the first patent for chewing gum [7]. The cosmetic industry market regulated by the USFDA is the most affected amongst any other markets [8]. A founding member of the US Society of Chemists, Dr. Raymond Reed was the first one to coin the term "cosmeceuticals" which denoted cosmetics with a therapeutic effect [8]. Topical tretinoin was the first cosmeceutical which was sold as a prescription item for treating appearance issues [9].

\section{Medicated Chocolate}

Chocolate is a widely accepted product which can be moulded in different ways to create a different taste and texture [10]. Cocoa which is the main ingredient in chocolate consists of various compounds like polyphenols, flavonoids, catechins, epicatechins etc. These properties of chocolate make it suitable for use as DDS. Bitter tasting drugs can ideally be incorporated into chocolates. Such a delivery system which consists of drugs incorporated in chocolate is called as a medicated chocolate. Chocolate DDS has many advantages such as bypassing first- pass metabolism and pre-systemic elimination in the GI tract. Also, chocolate is resistant to microbial growth and also does not allow the hydrolysis of water sensitive drugs [11]. Many drugs like chlorpheniramine maleate and cefdoxime protexil have been formulated as medicated chocolates. In a simple method of preparation, first the sugar and water are mixed and kept in an oven heated at 500C for 4-5 mins. Then cocoa butter is heated in the oven for a minute. Then the sugar syrup is removed from the oven and to it cocoa powder is added and the mixture is cooled. After this flavour is added to the prepared chocolate base for preparing the medicated chocolate, the prepared chocolate base is again heated in an oven at $50 \mathrm{o}$. The heating is continued until a free-flowing liquid is obtained. To this the drug is added and stirred on a magnetic stirrer to ensure uniform mixing.
This mixture is then poured in a polycarbonate mould and kept in the refrigerator for 15 mins until it solidifies [12]. An important aspect while formulating medicated chocolate is the addition of an adsorbent which is dicalcium phosphate. Dicalcium phosphate can be added to the formulation to ensure proper solidification [11].

\section{Evaluation parameters for medicated chocolate}

General appearance: The colour, odour, taste, texture of the medicated chocolate is evaluated since these are important parameters which decide the patient acceptability of the formulation.

Dimensions: The thickness, height and the diameter are measured using vernier callipers.

\%Moisture content: Moisture content can be determined using digital Karl Fischer titrator. The moisture content was calculated using the formula [13]:

Water $=$ [Volume $(\mathrm{ml})$ TS of water determination consumed $\times \mathrm{f}$ $(\mathrm{mg} / \mathrm{ml}) /$ weight of sample $(\mathrm{mg})] \times 100$

Where,

$\mathrm{f}=$ Number of $\mathrm{mg}$ of water corresponding to $1 \mathrm{ml}$ of water

TS=Test Sample.

Blooming test: Fat bloom is caused by the recrystallization of fat on the chocolate layer which gives it an unacceptable look [13].

Sugar bloom: The moisture in the chocolate formulation dissolves the chocolate in it. After evaporation of the water, the sugar gets recrystallized onto the surface of the chocolate. Each specimen subjected to treatment cycles contained:

- $\quad 30^{\circ} \mathrm{C}$ for $11 \mathrm{~h}$,

- Temperature moving for 60 mins,

- $\quad 18^{\circ} \mathrm{C}$ for $11 \mathrm{~h}$, and

- $\quad$ Temperature moving for 60 mins.

A test chocolate detailing saw, after the progression at $18^{\circ} \mathrm{C}$ for 11 $h$, regardless of whether or not blooming has occurred [13]

In vitro drug release study: For this study, USP dissolution apparatus type 1 was used containing $0.1 \mathrm{~N} \mathrm{HCl} .900 \mathrm{ml}$ of $0.1 \mathrm{~N} \mathrm{HCl}$ is filled in the vessel and the temperature is maintained at $37 \pm 0.5^{\circ} \mathrm{C}$ and $50 \mathrm{rpm}$. The medicated chocolate is placed in the dissolution medium and samples are withdrawn at regular time intervals. The samples are analysed using spectrophotometry [13].

\section{Medicated Chewing Gum}

The European Pharmacopoeia and the Committee for Medicinal products for human use defined medicated chewing gum (MCG) as "solid single dose preparation with a base consisting mainly of gum that are intended to be chewed but not swallowed, providing a slow and steady release of the medicine contained" [14]. Medicated chewing gums have several advantages over the conventional DDS i.e. the drug release can be controlled, they provide a faster onset of action, avoidance of first-pass metabolism [14]. The medicated chewing gum consists of a core composed of gum which may be coated. The gum contained in the core is an aqueous and insoluble gum which is mixed with sweeteners and flavours. The drug can be incorporated into the core [15] and chewing gums include metoclopramide [16], domperidone maleate [14], KSL (antimicrobial decapeptide) [17], nicotine [18] etc. 
There are several methods to prepare the medicated chewing gum. The first one is the conventional method in which a kettle is taken in which the melted gum base is added and to it other excipients like sweeteners and active ingredient are added. This mixture is then sent through rollers to obtain a thin ribbon. During this process, a light coating of powdered sugar is applied to avoid sticking of the gum and also to enhance the flavour. At a controlled temperature, the gum is allowed to cool for $48 \mathrm{~h}$ which sets the gum properly. The desired size of the gum is then cut [19]. The second method is the cooling, grinding and tabletting method. The gum base is cooled until it becomes brittle and the brittleness persists during grinding. The coolants used for cooling include liquid nitrogen, hydrocarbon slush, and solid carbon dioxide. Minute fragments of the cooled mixture is obtained by crushing or grinding. Then the coolant is removed by evaporation. The binders, coating agents, lubricants are then added to the powder so obtained and then compression is carried out by using punching [19]. Direct compression is another method for preparing medicated chewing gum. Here the co processed gum materials available in the market are directly compressed [19].

\section{Evaluation parameters for MCG Uniformity of content}

The content of randomly selected MCG is measured. It complies with the test if the content of each single MCG is between $85 \%$ and $115 \%$ of the average content [20].

\section{Uniformity of mass}

Weight of twenty MCG's selected at random is taken out of which not more than two single mass should vary the average mass [20].

\section{Stickiness}

The MCG's are placed on a planar surface on which a cylindrical hammer collides for a period of 10 mins. The sticking of mass to the hammer after 10 mins is observed and reported [21].

\section{In vitro drug release study}

In vitro drug release testing apparatus described in Pharmacopoeia for tablets and capsules is not suitable for testing medicated chewing gum. The in vitro drug release apparatus for the medicated chewing gum is designed so as to simulate the chewing process which takes place in the mouth. This apparatus consists of two pistons or jaws. The pistons are placed one on each side and when they move towards each other the chewing gum is pressed $[7,22]$. But this apparatus shows many disadvantages such as it does not exactly simulate the human chewing behaviour. While chewing the salivary secretion varies as the $\mathrm{pH}$ of the saliva also varies. In a study conducted on 3TabGums, a panel of volunteers were made to chew the gum in order to study the drug release characteristics of the gum [23].

\section{Medicated Lipstick}

Lipstick is among the widely used lip care cosmetic by women which is available in different colours and designs. Lipstick consists of a waxy base into which the colour is incorporated. Medicated lipsticks in addition to this also contain an active ingredient. The drugs used in the formulation of medicated lipsticks include salicylic acid [24], allantoin [25], terbinafine hydrochloride [26], acyclovir [27] etc. Medicated lipsticks can be used to treat different lip problems like infection, lip dryness, swelling etc. [27].

Attempts have been made to incorporate antiviral drugs like acyclovir into lipstick for the treatment of herpes labialis, a viral disease. Topical application of acyclovir leads to evaporation of the active ingredient from the surface of the skin and hence a more effective way of topical delivery of acyclovir was made by incorporating acyclovir into lipstick formulation. Acyclovir-loaded microsponges were prepared which were then incorporated into the molten wax containing colour [27]. In another study, medicated lipstick containing allantoin was made which replaced synthetic vehicles with honey and ghee. The evaluation studies reported that the lipstick base made using natural vehicles was satisfactory in terms of hardness and spreadability. The medicated lipstick was also reported to have satisfactory hardness, spreadability and smoothness [25].

\section{Evaluation parameters for medicated lipstick}

Melting point: Lipstick sample filled in a capillary tube sealed on both ends is fastened to a thermometer. The whole assembly is to be dipped into a beaker full of water which is heated with continuous stirring. The melting point is the temperature at which the material moves along through capillary tube [26].

Hardness test: Lipstick is held horizontally at a certain distance from the edge of the support. The weight is gradually increased by a specific value at a certain time interval. The hardness depends on the weight at which the lipstick breaks [28].

Medicated tattoo: Transdermal DDS represents a painless and convenient mode of drug delivery as compared to the conventional DDS [29]. Medicated tattoo is a recent advanced technology to improve transdermal drug delivery. Medicated tattoo is similar to the temporary tattoo applied on the skin for beautification purpose except that it contains an active ingredient intended for therapeutic effect [30]. Currently the drugs incorporated in medicated tattoos are acetaminophen and vitamin C produced by Lipper-Man Ltd [31].

Patented work on medicated tattoo revealed that $3 \mathrm{M}$ components such as backing membrane, drug reservoir, controlled release membrane and skin adhesive are a part of $75 \%$ of the transdermal patches in the world [4]. In the same study, medicated tattoo was prepared which consisted of seven layers i.e. the adhesive layer, drug substance layer, coloured layer, clear base, release base and base paper. The drug substance can be incorporated into the drug substance layer or it can be mixed with the skin adhesive and incorporated into the adhesive layer. The conventional tattoos were then printed using offset lithography and silk screen techniques. Another patented work on medicated tattoos included preparation of cosmetic skin tattoo for hiding skin imperfections such as blemishes, age marks etc. which included incorporation of an antibacterial agent [32]. The medicated tattoo is worn on the skin in the same manner in which a temporary tattoo is worn. The advantages include ease of termination of therapy. For the termination of the therapy, the colour of the tattoo can be compared with that of the colour on the colour chart provided by the manufacturer [31].

\section{Medicated Kajal}

Kajal or kohl is a cosmetic which is used since ancient times. It has been used widely due to its benefits to the eyes [33]. Medicated kajal is thick and black in colour in which a drug is incorporated for therapeutic effect. Attempts have been made to incorporate herbal drugs like Eclipta alba and Vernonia cinerea into the kajal base [34]. Eclipta alba is known to have antimicrobial and antiinflammatory properties [35]. Vernonia cinerea consists of triterpenes whose antimicrobial and anti-inflammatory activity has been reported $[34,36]$. In this study the extract or juice of the two herbs was prepared. A cloth was soaked in 
the juice until the juice was exhausted. The cloth was then lighted in a mud lamp. It was collected in a clean and dry plate and then the powder so obtained was mixed with coconut oil to be used as medicated kajal [34]. Shashikant et al. developed an ocular cosmeceutical of sulfacetamide for treating eye infections and evaluated its potential for ocular drug delivery. The in vitro drug release studies were carried out using dissolution apparatus which simulated the eye conditions. Based on this study they concluded that the kajal which was developed as a cosmeceutical can treat eye infections if applied three times a day as well as it can be used to give an aesthetic appeal to eye [37].

\section{Medicated Bindi}

Medicated bindi is essentially a transdermal patch with a drug to be worn on the forehead. Drugs like iron and iodine have been used in preparing medicated bindis. In one of the study involving preparation of iodine bindis, a $2 \%$ solution of iodine was used. The prepared iodine solution was mixed with the polymers i.e. ethyl cellulose and PVP and plasticizer i.e. propylene glycol and this solution was casted on a petri plate to form a film.

\section{Evaluation parameters for medicated bindi}

Thickness: The thickness test is performed by measuring the thickness of the patch at five different places using a micrometer screw gauge or Vernier callipers and then calculating the average.

Folding endurance: Repeated folding of the patch at the same place until it breaks determines the folding endurance of the patch.

Content uniformity: A certain area of the film is to be taken which contains a certain amount of drug which is to be dissolved in phosphate buffer with $\mathrm{pH}$ 7.4. The sample is to be further diluted, filtered and analysed by UV-Spectrophotometer.

In vitro drug release study: Patches are to be placed in a beaker and care needs to be taken that the patches are secured firmly to the beaker. Phosphate buffer saline is added to the beaker as a dissolution medium and this assembly is to be placed on the magnetic stirrer. At specific time intervals, aliquots are taken using syringe and sink condition is to be maintained. The samples are filtered and concentration of drug is determined by measuring the absorbance of the drug [38].

\section{Medicated Nail Lacquer}

Many studies conducted provide evidence that the human nail behaves more like a hydrophilic gel membrane unlike the stratum corneum which is a lipophilic membrane [39]. Permeability through the nail is relatively less and only a limited number of drugs pass through the nail. Hence for the drug to pass through the nail plate, it should have a small size and should be available in non-ionic form [40]. Among the various treatment options available to treat nail infections, medicated nail lacquer is a preferred new method. Drugs such as ciclopirox and amorolfine have been formulated as nail lacquers for topical delivery to the nail. After application of the nail lacquer, a film is formed over the nail plate through which the drug is released slowly allowing sustained diffusion of the drug [41]. Though the medicated nail lacquers have high patient acceptability, there are certain limitations associated with it. The most important limitation to be considered is low permeability of the nail plate. This lead to emergence of newer techniques for increasing penetration of drugs through the nail plate. Vanstone etal. used laser poration technique for controlling drug delivery through the nails [42]. They concluded that complete poration of the nail led to increase in the transport of the drug into the nail whereas partial poration enabled controlled drug delivery through the nails. In another study conducted by Hao et al. iontophoresis was used to enhance delivery of ciclopirox through the nails [43]. In this study it was concluded that constant voltage iontophoresis significantly enhanced the penetration of ciclopirox into and across the nails.

\section{Evaluation parameters for medicated nail lacquer}

Drying time: A specific amount of the nail lacquer is applied on a specific area of a glass plate. The time required for complete drying of the film is the drying time [41].

Non-volatile content: The sample is evenly spread on a glass petri dish and placed in the oven at a specific temperature and time. The difference in the weight of the sample before and after drying is the non-volatile content [44].

\section{Medicated Hair Styling Gel}

Hair styling gel is a hair cosmetic which can be applied on damp hair to give them a lift from the scalp. Hair styling gels can be used in individuals with diffuse hair thinning as these provide resemblance to voluminous hair [45]. Now-a-days, medicated hair styling gels have been used in the treatment of dandruff. Dhamane et al. formulated an antidandruff hair gel of fluconazole and tea tree oil for the treatment of seborrheic dermatitis. In the study, triethanolamine was used as a gelling agent. Three formulations were prepared in which one formulation contained both fluconazole and tea tree oil, another contained only fluconazole and the third one contained tea tree oil. The three formulations were evaluated for their efficacy. They reported that the formulation containing fluconazole and tea tree oil as well as the one containing only tea tree oil showed higher anti-fungal activity [46]. Nayak et al. conducted a study on anti- dandruff hair styling gel of ketoconazole. Three formulations containing $0.5 \%, 1 \%$ and $1.5 \%$ of ketoconazole were prepared and evaluated for their anti-fungal activity. The study suggested that hair styling gel containing $1 \%$ of the drug was more effective than the other two formulations because of effective release and attainment of minimum inhibitory concentration of ketoconazole [47].

\section{Evaluation parameters for medicated hair styling gel}

Physical examination: The colour, clogging, presence of air bubbles and homogeneity is examined.

\section{$\mathbf{p H}$ : The $\mathrm{pH}$ is examined using digital $\mathrm{pH}$ meter or $\mathrm{pH}$ paper.}

Viscosity: The viscosity can be evaluated using Brookfield viscometer and the corresponding viscosity and torque values are noted down.

Spreadability: The gel is spread between two glass slides and the slide on the top is pulled using a string attached to a hook and the time required for the glass slide to cover a specific distance is noted. The spreadability can be calculated by using the formula:

\section{$\mathrm{S}=\mathrm{ML} / \mathrm{T}$}

Where, $\mathrm{S}=$ Spreading coefficient, $\mathrm{M}=$ Weight tied to upper slide, $\mathrm{L}=$ Length of slides, $\mathrm{T}=$ Time taken to separate the slides.

In vitro anti dandruff activity: The agar well diffusion method is used for the assessment of anti-dandruff activity. The test microorganism used is Malassezia furfur. The sub-cultured microorganisms are streaked on agar plates and then the plates are incubated and the zone of inhibition is recorded [46]. 


\section{Medicated Hair Conditioner}

Hair conditioners provide softness and glossiness to the hair. They have also been found to be effective in reconditioning the hair post treatments like waving, straightening and colouring. Hair conditioners thus reduce friction among the hair shafts which would otherwise lead to tangling of hair [48]. Medicated hair conditioner can be used to modify the look of the hair as well as to combat hair conditions like seborrhoea. Patented work by Selega et al consists of medicated hair conditioner as a formulation to treat seborrhoea. In their study, anhydrous sulfur was used as a medicament to treat seborrhoea. Organically modified hectorite clay was used as gellant which helps the composition to retain one phase. Also, the setting test was performed on the prepared formulation to check whether the sulfur crystallized or settled during heating after the formulation was made. Their study suggests that no such crystallization or settling of sulfur occurs [49].

\section{Wearable Earring}

Many advances are going on in the field of smart wearable systems. These smart wearable systems have the capability of responding to the patient and his environment and also can collect huge amount of biomedical information from the individual using it [50]. Smart wearable systems form an important part of health tracking. Smart earrings are one of the several products in which sensors are used. These sensors have the capability to detect heartbeat, calories burned etc. [51]. The possibilities of placement of wearable biosensors can be many. These can be worn on several parts of the body like wrist, ankle, ears etc. [52]. The use of wearable technology in the field of cardiology is also increasing. Integrated and wearable earring as biosensor for troponin detection has been reported (Figure 2). The earring is meant to be worn on the earlobe as a biosensor. The wearable earring consisted of a silicon wire and a substrate. The silicon wire was functionalized with monoclonal antibodies for detection of troponin. When positive signal is received from the biosensor, the release of the drug from the microneedle patch work as a wristband is triggered [53,54]. In another study Alam et al. designed a wearable smart earring which can differentiate activities such as coughing, yawning and deglutition [55]. According to them, these activities help in identification of physiological symptoms and chronic psychological conditions which are not directly observable from daily activities. They concluded that this wearable device needs more miniaturization and light weight conversion for it to be used as a wearable earring for older adults (Figure 3 ).

\section{Epi-Bracelet}

Epi-bracelet is a type of an epinephrine auto-injector which is portable and small [56]. It is intended to be used in treatment of anaphylaxis. It is one of the few products which is on the horizon in the United States [56]. Auto injectors currently available can be used for the treatment of various conditions like anaphylaxis, diabetes mellitus, growth hormone deficiencies etc. [57]. The first epinephrine autoinjector which was called the "EpiPen" was approved by the FDA in 1987. But very recently it has been recalled by Mylan due to device activation failures [57].

Michael Langan was the first person who filed a patent on Epibracelet in the year 2011. He had designed an epinephrine autoinjector which also served as a fashion accessory [58]. This bracelet worked in three simple steps i.e. Twist, Turn and Press. The device is twisted 180 degrees to form an 'S' shape after which the bracelet which forms two semi-circular arcs locks in place and the trigger mechanism is activated. For injecting the dose from the bracelet, the cap of the medication cartridge needs to be rotated 120 degrees. The semi-circular arc is pressed against the thigh or shoulder and the dose of epinephrine is delivered. The needle length of the injector varies according to the dose. For $0.15 \mathrm{mg}$ of the dose, the needle length is 2 centimetres and for $0.3 \mathrm{mg}$ of the dose, the needle length is 3 centimetres (Figure 4).

\section{Polymers used in different DDSs}

Selection of polymers depends on the type of DDS. Medicated chewing gum base composition is an important factor on which the perception of flavour while chewing depends [59]. Polymers are also used in the fabrication of medicated tattoo. Sustained release of medicaments from medicated tattoo is achieved by using microencapsulation or hydrogel polymers [4]. Similarly, when the coating of medicated nail lacquer is desired to remain on the nails for an extended period of time, hydrophilic polymers can be used. Briedis et al. studied the effect of three different film forming polymers on the release of naftifine hydrochloride containing medicated nail lacquer [60]. They concluded that Eudragit RL 100 and Eudragit RS 100 were suitable polymers to be used in the medicated nail lacquer whereas ethyl cellulose could not be used as a film former in the nail lacquer as it showed poor solubility in the mixture of organic solvents used. The drying time was also more as compared to the other formulations and the film formed after drying was observed to be sticky [60]. The following table summarizes the different types of polymers used in different DDS (Table 1).

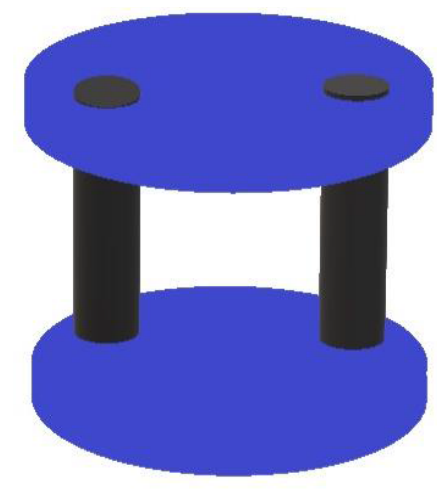

Figure 3: Wearable earring with silicon wire.

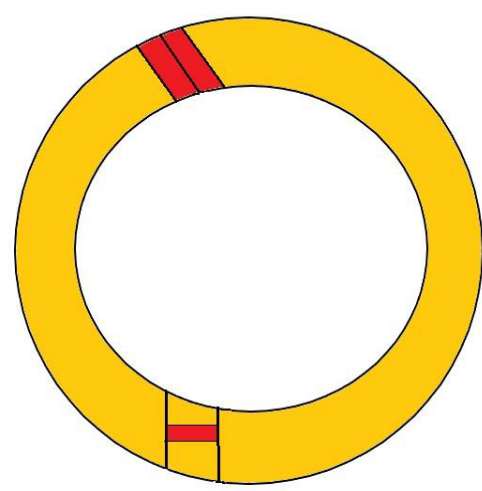

Figure 4: Epibracelet. 


\begin{tabular}{|l|l|}
\hline Type of DDS & Polymers used \\
\hline Medicated chewing gum & $\begin{array}{l}\text { Co-polymers of styrene-butadiene, polyethylene, } \\
\text { polyisoprene etc. }\end{array}$ \\
\hline Medicated tattoo & Ethyl cellulose, starch, hydroxypropyl methyl cellulose \\
\hline Medicated bindi & Ethyl cellulose, polyvinylpyrrolidone \\
\hline Medicated nail lacquer & Nitrocellulose, eudragit, ethylcellulose \\
\hline
\end{tabular}

Table 1: Polymers used in different types of DDS

\section{Toxicity}

Toxicity of medicated chewing gum has not been reported elsewhere. The contents of the flavour should be checked by such individuals who are prone to allergy [61]. It has been found that sorbitol containing medicated chewing gum caused diarrhoea due to high osmotic pressure in the gastrointestinal tract. Imfeld et al. conducted a study on chlorhexidine chewing gum for four-week randomized, double-blind study on elderly patients with salivary hypofunction. They reported the appearance of light brown discolouration of the teeth [62]. Also, adverse effects of ciclopirox nail lacquer has been studied on patients which showed that such patients suffered with periungual erythema and erythema of the proximal nail fold. Similarly, local effects such as burning, pruritic and pain around the nail bed were reported with the use of amorolfine nail lacquer [63].

\section{Regulatory Aspects}

Injectors which are used in combination with a specified drug or biologic are considered as combination products under $21 \mathrm{CFR}$ 3.2(e). They are regulated on basis of their primary mode of action. Combination products which have the drug/biologic product primary mode of action are regulated by CDER or CBER. Only one marketing application i.e. Biologic License Application (BLA) or New Drug Application (NDA) is sufficient for combination products consisting of an injector and drug/biologic product combination. The NDA submission must include documentation describing that the drug or biologic is currently approved for marketing in the necessary configuration for use in the injector [64]. In case of cosmetics which also serve as drugs, they are formulated and marketed as cosmetics which also have additional benefits of a drug. In case of medicated chewing gums, they have to be manufactured under cGMP conditions and must comply with food chemical specifications and must be "generally regarded as safe". These are regulated by FDA title 21 CFR section 172.615 [60]. Also, the USFDA has given guidelines on the regulation of nail care products. According to the USFDA, nail products which are used to treat medical problems like nail fungus are regulated as drugs [65].

\section{Conclusions}

Elegant DDS enables drug delivery in a smart manner for beautification purpose. Although it has a potential to be used as a system of drug delivery, many issues are associated with it. As there is less research conducted in this area, there is no compendial method available for evaluation of some of these DDS. For instance, for in vitro drug release study of medicated chewing gum a different apparatus is designed but it not official. Similarly, wearable devices also have certain disadvantages. The patient must know the proper way to use the device. The patient might be self-harmed, if he/she does not use the device appropriately. There is also a possibility of spurious or substandard devices entering the market. This may adversely affect the patient's health. Much research is still to be carried out on such DDS intended for drug delivery and elegance or modification of looks.

\section{References}

1. Tiwari GR, Tiwari B, Bhati SL, Pandey S, Banerjee SK, et al. (2012) Drug delivery systems: An updated review. Int J Pharm Investig 2: 2-11.

2. Jain KK (2014) Current status and future prospects of DDS. Methods Mol Biol 1141: 1-56.

3. Chan M, Estève D, Fourniols JY, Escriba C, Campo E (2012) Smart wearable systems: Current status and future challenges. Artif Intell Med 56: 137-156.

4. Lipper C, Man M, Parisek CB (2001) Medicated tattoos.

5. Lippi D (2009) Chocolate and medicine: Dangerous liaisons? Nutrition 25: 1100-1103.

6. Sanares AME, King NM, Itthagarun A, Wong HM (2009) Chewing gum as a medium for the delivery of anticariogenic therapeutic agents: a review. Hong Kong Dental Journal. 6: 13-22.

7. Rassing MR (1994) Chewing gum as a DDS, Adv Drug Deliv Rev 13: 89-121.

8. Newburger AE (2009) Cosmeceuticals: myths and misconceptions. Clin dermatol 27: 446-452.

9. Draelos ZD (2009) Cosmeceuticals: undefined, unclassified, and unregulated Clin dermatol 27: 431-434.

10. Patel J, Joshi M, Thakkar V, Gohel M, Baldaniya L, et al. (2016) Medicated chocolate containing mcefpodoxime proxetil: a novel solid dosage form for paediatric patient. Proceedings of RK University's First International Conference on Research and Entrepreneurship.

11. Reddy S, Mounika K, Venkatesham A (2017) Design and fabrication of medicated chocolate formulation by chocolate DDS. Int $\mathrm{J}$ Curr Pharm Res 9: 128-133.

12. Sharma M, Jain DK (2012) Chocolate formulation as DDS for paediatrics. Indonesian J Pharm 23: 216-224.

13. Patel N, Diwan S, Shukla K, Tomar P, Jain H, et al. (2015) Chocolate Drug delivery system: a review. Indo Am J Pharm Sci 2: 1077-1081.

14. Paradkar M, Gajra B, Patel B (2016) Formulation development and evaluation of medicated chewing gum of anti-emetic drug. Saudi Pharm J 24: 153-164.

15. Jacobsen J, Christrup LL, Jensen NH (2004) Medicated Chewing Gum: Pros and Cons. Am J Drug Deliv 2: 75-88.

16. Grabnar I, Maggi L, Cocchietto M, Conte U, Voinovich D (2007) Bioavailability of metoclopramide from a new chewing gum device. $\mathrm{J}$ drug deliv sci and technol 17: 173-176.

17. Na DH, Faraj J, Capan Y, Leung KP, DeLuca PP (2005) Chewing gum of antimicrobial decapeptide (KSL) as a sustained antiplaque agent: preformulation study. Journal of Control Release 107: 122-130.

18. Cherukuri SR, Pinney JM, Henningfield JE, Sasan A, Cone EJ, et al. (2011) Medicated chewing gum delivery system for nicotine. US Patent 6: 344-222.

19. Gadhavi AG, Patel BN, Patel DM, Patel CN (2011) Medicated Chewing Gum-A 21st Century Drug Delivery Sysytem. Int J Pharm Sci Res 2: 1961.

20. Aslani A, Rostami F (2015) Medicated chewing gum, a novel DDS. J res med sci: the official journal of Isfahan University of Medical Sciences 20: 403-411.

21. Garg TK, Goyal A (2014) Medicated chewing gum: patient compliance oral DDS. Drug Deliv Lett 4: 72-78.

22. Kvist LC, Andersson SB, Berglund J, Wennergren B, Fors SM (2000) Equipment for drug release testing of medicated chewing gums. J Pharm Biomed Anal 22: 405-411.

23. Maggi L, Segale L, Conti S, Machiste EO, Salini A (2005) UConte, Preparation and evaluation of release characteristics of 3TabGum, a novel chewing device. Eur J Pharm Sci 24: 487-493.

24. Sathish S, Mahesh C, Saikat D, Lavanya V, Suresh B (2012) Preparation and evaluation of salicylic acid medicated lipstick, Journal of Advanced pharmaceutical Sciences 2: 289-94.

25. Shaikh S, Bhise K (2008) Formulation and evaluation of medicated lipstick of allantoin. Asian J Pharm. 2: 91.

26. Majumdar SF, Kakadiya BL (2015) Desiging medicated lipstick for anti-fungal therapy. Am J Pharm Res Vol: 5. 
27. Yadav $P$, Nanda S (2014) Development and evaluation of some microsponge loaded medicated topical formulations of Acyclovir. Int J Pharm Sci Res 5: 1395-1410.

28. Munawiroh SZ, Nabila AN, Chabib L (2017) Development of Water in Olive Oil (W/O) Nanoemulsions as Lipstick Base Formulation. Indian Journal of Pharma Medicine and Biological Sciences 6: 37-42.

29. Lee H, Song C, Baik S, Kim D, Hyeon T, et al. (2017) Device-assisted transdermal drug delivery. Adv Drug Deliv Rev 17: 30180-30181.

30. Sahoo CK, Nayak PK, Sahoo TK, Dasari P, Dandamundi S (2011) A Review of transdermal DDS. Journal der PharmazieForschung 2: 32-51.

31. Kumar R, Philip A (2007) Modified transdermal technologies: Breaking the barriers of drug permeation via the skin. Trop J Pharm Res 6: 633-644.

32. Muratore-Pallatino J, Hasley M, Hasley MM (2002) Cosmetic skin tattoo. U.S. Patent Application 10: 656

33. Chaudhri SK, Jain NK (2009) History of cosmetics. Asian J Pharm 3: 164.

34. Dang R, Das K (2015) Total safety management through standardization of formulated ayurvedic Kajal using Eclipta alba and Vernonia cinerea herbs. World Sci News 5: 32-44

35. Neeraja PV, Margaret E,Alba E (2012) Hassk: a valuable medicinal herb. International Journal of Current Pharmaceutical Review and Research 2: 188197.

36. Shelar D, Tikole S, Kakade T (2014) Vernonia cinerea: a review. J Curr Pharm Res 4: 1194-1200.

37. Shashikant YA, Deepak GY (2013) Kajal: Cosmeceutical. Int J Pharm Arch Vol: 2.

38. Jagtap SB, Sawant DA, Jadhav AR, Choudhari M (2017) Formulation and Evaluation of Transdermal Patch of lodine as Ladies Bindi. Int J Chemtech Res 10: 90-101.

39. Rajendra VB, Baro A, Kumari A, Dhamecha DL, Lahoti SR et al. (2012) Transungual drug delivery: An overview. J Appl Pharm Sci 2: 203-209.

40. Kumar PT, Raju PN (2013) Transungual drug delivery: a promising route to treat nail disorders. Int J Pharm Res 2: 22-23.

41. El-sherif NI, Sharma RN, Abdelbary G (2018) In-situ gels and nail lacquers as potential delivery systems for treatment of onychomycosis. A comparative study. J Drug Deliv Sci Technol 43: 253-261.

42. Vanstone S, Cordery SF, Stone JM, Gordeev SN, Guy RH (2017) Precise laser poration to control drug delivery into and through human nail. J Control Release 268: 72-77.

43. Hao J, Smith KA, Li SK (2009) lontophoretically enhanced ciclopirox delivery into and across human nail plate. J Pharm Sci 98: 3608-3616.

44. Kurian N (2017) Formulation and evaluation of transungual drug delivery of fluconazole using permeation enhancers screened by hydration of nail plate, research and reviews. A Journal of Pharmaceutical Science 8: 32-45.

45. Madnani N, Khan K (2013) Hair cosmetics. Indian J Dermatol Venereol Leprol 79: 654-667.
46. Dhamane SP, Tayade NV, Potnis VV, Kulkarni AS, Gadekar AS (2015) Formulation and evaluation of antidandruff hair gel for treatment of seborrheic dermatitis. World J Pharm Res 4: 1260-1271.

47. Nayak SH, Nakhat PD, Yeole PG (2005) Development and evaluation of cosmeceutical hair styling gels of ketoconazole. Indian J Pharm Sci 67: 231.

48. Bolduc C, Shapiro J (2001) Hair care products: waving, straightening, conditioning, and coloring. Clin Dermatol 19: 431-436.

49. Selega ZE, Khalil EN, McKavanagh GM (1980) Johnson Products Co Inc Medicated hair and scalp conditioner. US Patent 4: 237-112.

50. Chan M, Estève D, Fourniols JY, Escriba C, Campo E (2012) Smart wearable systems: Current status and future challenges. Artif Intell Med 56: 137-156.

51. Vu T, Khoa A (2015) Wearable Smart Technologies: New Ear of Technology.

52. Appelboom G, Camacho E, Abraham ME, Bruce SS, Dumont EL, et al. (2014) Smart wearable body sensors for patient self-assessment and monitoring. Arch Public Health 72: 28.

53. Kong T, Su R, Zhang B, Zhang Q, Cheng G (2012) CMOS-compatible label-free silicon-nanowire biosensors to detect cardiac troponin I for acute myocardial infarction diagnosis. Biosensors and Bioelectronics 34: 267-272.

54. Zheng Y, Chan CK, Li Y, Poon CC, Zhang Y (2021) Health Informatics: From Point-of- Care Bio-sensing to Wearable Sensing for p-Health, Transactions of Japanese Society for Medical and Biological Engineering 50: 510-521.

55. Alam MAU, Roy N, Gangopadhyay A, Galik E (2017) A smart segmentation technique towards improved infrequent non-speech gestural activity recognition model. Pervasive and Mobile Computing 34: 25-45.

56. Greenberger PA, Wallace DV, Lieberman PL, Gregory SM (2017) Contemporary issues in anaphylaxis and the evolution of epinephrine autoinjectors: What will the future bring?. Ann Allergy Asthma Immunol 119: 333-338.

57. Vadlapatla R, Wong EY, Gayakwad SG (2017) Electronic DDSs: An overview. J Drug Deliv Sci Technol 41: 359-366.

58. Langan ML (2012) Automatic Medication Injection Device. WO/2012/058192.

59. Konar N, Palabiyik I, Toker OS, Sagdic O (2016) Chewing gum: Production, quality parameters and opportunities for delivering bioactive compounds. Trends Food Sci Technol 55: 29-38.

60. Šveikauskaitè I, Briedis V (2017) Effect of Film-Forming Polymers on Release of Naftifine Hydrochloride from Nail Lacquers. Int J Polym Sci.

61. Patel CJ, Asija S, Patel P, Dhruv M, Tyagi S (2012) Medicated chewing gum: a modern era of novel DDS. Research Journal of Pharmaceutical Dosage Forms and Technology 4: 293-299.

62. Imfeld T (2006) Chlorhexidine-containing chewing gum. Schweiz Monatsschr Zahnmed 116: 476-483.

63. Shirwaikar AA, Thomas T, Shirwaikar A, Lobo R, Prabhu KS (2008) Treatment of onychomycosis: an update. Indian journal of pharmaceutical sciences 70 : 710.

64. https://www.fda.gov/combinationproducts/aboutcombinationproducts/ ucm101496.html/

65. https://www.fda.gov/cosmetics/productsingredients/products/ucm127068.htm/ 